\title{
ULTRSOCIALITY, GOODS THEORY, AND PRIMITIVE AGRICULTURE IN COSMOPOLITAN EARTH AND PUTATTIVE EXTRATERRESTIAL MICROBIAL SYMBIONTS
}

\author{
Kevin B. Clark, Ph.D. \\ (kbclarkphd@yahoo.com and kevin.clark@mpg-alumni.de)
}

Director, Felidae Conservation Fund, Mill Valley, CA 94941, USA; Co-Chairperson, Science Advisory Board, Cures Within Reach, Chicago, IL 60602, USA; Domain Champion in Biomedicine and Campus Champion, NSF Extreme Science and Engineering Discovery Environment (XSEDE), National Center for Supercomputing Applications, University of Illinois at Urbana-Champaign, Urbana, IL 61801, USA; Affiliate, NASA Ames Research Center, Mountain View, CA 94035, USA; Affiliate, NASA NfoLD, NASA Astrobiology Program, NASA Ames Research Center, Mountain View, CA 94035, USA; Advisor, Universities Space Research Association, Columbia, MD 21046, USA; Subject Matter Expert and Mentor, Penn Center for Innovation, University of Pennsylvania, Philadelphia, PA 19104, USA; Consultant and Collaborator, Research and Development Service, Veterans Affairs Greater Los Angeles Healthcare System, Los Angeles, CA 90073, USA; Main Organizer, Shared Interest Group for Natural and Artificial Intelligence (sigNAI), Max Planck Alumni Association, 14057 Berlin, Germany; Member, Nanotechnology and Biometrics Councils, Institute for Electrical and Electronics Engineers (IEEE), New York, NY 10016-5997, USA.

White Paper Running Head: ClarkKevinB2, Ultrasociality in Earth and Extraterrestrial Microbes 


\begin{abstract}
Farming microbes select vital tradeoffs favoring economics-reliant ultrasociality, a superior collective state derived from multilevel sociogenetic effects. This public-goods interpretation yields better insights into sociobiological transitions on Earth and model habitable worlds, including consumer drivers of life histories and biospheres.
\end{abstract}

\title{
BACKGROUND
}

Goods theory remains a popular tool for studying and describing competitive and cooperative microbial behaviors across differing ecological situations (Box 1). But, where lie the limitations of public goods theory for explaining microbial sociobiology? Rauch et al. (24), for example, importantly find both fixed and conditional investment strategies beat public goods game dilemmas for microbes confronting eco-evolutionary tradeoffs that threaten long-term viability of predominant cooperator subpopulations against their freeloader rivals. The authors generalize the outcomes of their yeast-sucrose model system as a predictable case from public goods game theory, where the ability to regulate economic investments through gene-supported smart or rational social strategies allow organisms to colonize more challenging environments over short to long timescales. While these conclusions certainly offer insights into the fundamental nature of social conflict instigation and resolution, Rauch et al. (24), among other scientists, fail to confront severe trending criticisms concerning the value of public goods theory for understanding broader contexts of complex social microbial behavior (e.g., 23,30,31), including possible farming-unique ultrasociality and the use of fallible rational and irrational economicbased decisions to maximize generational and transgenerational outcomes for survival and reproduction. If found to be powerful and internally and externally valid within and across microbiota systematics, such consumer-based effects may be expected to play major roles in shaping the life histories of microbes and the structures and processes of microbial communities, with particular dramatic influence over the origins and evolution of niches to biospheres for habitable worlds beyond Earth. Unfortunately, the sociobiology of early life, or life at any stage of evolution and development for that matter, is often given little to no scientific significance by astrobiologists. Future unmanned and manned exploration of our solar system and elsewhere in the universe will give unprecedented opportunities to appraise contemporary sociobiological thinking and to apply those foundational efforts to practical empirical and theoretical investigations into the origins and evolution of life and societies.

\section{ECONOMICS STRUCTURE OF ULTRASOCIALITY}

Ultrasociality, a sophisticated form of mass social organization dependent on division of labor and public-goods-based agricultural subsistence (cf. 17), is considered the evolutionary pinnacle of social-group expression, with robust complex contributions from genes, culture, and other motivating factors. Despite innovative social organization and competencies of simple animal and nonanimal life, sociobiologists rarely credit ultrasociality to clades of organisms older than the Ecdysozoa-Lophotrochozoa divergence $(17,25)$, believing ultrasociality is exemplified by humans and few other members of Kingdom Animalia. Nevertheless, a major microbiota taxon Dictyostelids - produce complex cosmopolitan societies exhibiting essential traits of ultrasociality, including biomass proliferation, habitat diversity and dominance, sophisticated 


\section{Box 1. Forecasting the Economic Origins of Microbial Ultrasociality}

Application of economics theory in sociobiology continues to garner strong interest among scientists as a means to explain and predict the evolution and behavior of all social microbiota. The basis of economics theory involves the types and exchange of goods supplied and demanded. Goods may fall within categories for private, club, common-pool, and public resources and (commoditized) services. Private and club goods are associated with property owner rights or some level of excludability, whereas common-pool and public goods are nonexcludable. Scarcity of goods motivates rivalry between consumers and may influence acquisition of excludable private goods and non-excludable common-pool goods. Club and public goods are non-rivalrous or minimally rivalrous properties. Scientists for decades have developed and presented arguments of varying soundness or cogentness for excludable/nonexcludable and rivalrous/nonrivalrous microbial societies. Trends in empirical observations for public and private goods behavior in microbes suggests that multilevel selection pressures forced individual-group tradeoffs responsible for convergent evolution of ultrasocial life and sustained elevated viability over both evolutionary and ecological time periods. Microbes displaying these characteristics offer good models to study further the ecoevolutionary complexity of societal formation, stability, and transition, perhaps giving opportunities to reveal similar (or dissimilar) eco-evolutionary processes occurring at other locations in the universe.

division of labor, kinship-(in)dependent conflict instigation/mediation, and tendencies of managed livestock-/crop-like farming $(4,5,29,33)$. Appreciation of near ultasocial microbial characteristics puts all animal-centric views of ultrasociality evolution into a necessary larger frame. Without such details, scientists arguably cannot fully explain multilevel sociogenetic selection pressures that force individual-group tradeoffs and convergent evolution of ultrasociality and other social forms within and across systematics (cf. 21.22).

An example may be developed from Thutupalli et al. (31), who conclude the nematode Caenorhabditis elegans farms soil bacteria in a qualitative different manner than behaviorally unsophisticated microbial systems, which, with possible exception of the cellular slime mold Dictyostellium discoideum, depend on metabolic costs of goods synthesis, such as that used by the fungus Saccharomyces cerevisiae (24), and not exploration-exploitation strategies and other phenomena. Using related claims, some scientists now judge instances of microbial sociality as illusory outcomes of life-history tradeoffs and nonsocial multiple fitness traits selected by deterministic or stochastic eco-evolutionary pressures $(23,30)$. Such claims strongly contest the power and validity of applying public goods game theory to microbe-salient scenarios, including that espoused by Rauch et al. (24), where regulation of economic investments through genesupported fixed or conditional social strategies permits microbes to colonize exigent timevarying environments. But, in reaching their conclusions, Thutupalli et al. (31) and additional scientists avoid a significant opportunity to elaborate and strengthen their too narrow animalcentric arguments on evolved public-goods-dependent social structure and function - an outcome that directly impacts scientific interpretations of ultrasociality evolution.

\section{ECONOMICS-DRIVEN EMERGENCE OF PRIMITIVE MICROBIAL AGRICULTURE}


Similar to ultrasocial ants and termites (17), sociogenetic features of adaptive microbial symbioses promote economics-dependent ultrasociality in caste-like cosmopolitan (super)colonies. Experimental findings confirm different microbe types, via single or multiple gene mutations, phenotypic switching, and learned behavioral modification (4,5,25,29), can alter their social status within a colony. Social mobility, induced by supply-stressed environmental resources and cell-cell communications reporting community-transformation needs, compels management of individual-group tradeoffs to optimize survival and reproduction. Sometimes individual or group goals are achieved through ruthless, selfish Machiavellian-type rivalry and deception and other times more peaceable, honest altruistic actions are taken (e.g., 1,2,26,32). Whether through these sorts of reciprocating, policing, or alternative social constraint methods, microbes demonstrate remarkable aptitudes to harness intracolony resources to cope with their niche and to conquer additional ones, as observed for microbes transitioning from hunter-/gathersubsistence (e.g., predation, phototrophism, free-nutrient absorption) to (tended/untended) agrarian-type communities that help direct group versatility toward ultrasociality $(4,5,28,33)$.

Making farming-based economics a criterion for ultrasociality, as done for animals (17), requires special attention to $D$. discoideum primitive agriculture $(4,5,29,31,33)$, which evolved as a clonespecific trait that influences life history and fitness. While these microbes and possibly additional ones surely cannot match the farming technology and financial market sophistication of humans, their prowess to survive, reproduce, and establish kin-dependent niche dominance through complex behaviors and exploration-exploitation tradeoffs should not be underestimated. $D$. discoideum farmers proliferate in ecological conditions of low nutrient availability, when solitary hunting is abandoned and ordered motile social collectives, called slugs, are formed to begin fruiting body differentiation and sporulation. Slugs comprised of farmers, as compared to nonfarmers, migrate shorter distances to relocate in more favorable, if imperfect, ecological settings as well as prudently harvest bacteria to reserve stores for later consumption during codispersal. The symbiosis between farmer and bacterial livestock is furthermore selective. Farmer amoebae carry proportionately higher populations of preferred-eating bacteria, with additional mixed populations of herd-dog bacteria to help secure and privatize livestock. Taken together, the qualities of $D$. discoideum primitive agriculture support dynamic consumer marketplaces, with structural and operational capabilities of kinship groups effecting sound trading partner choices, creation of strong local business connections, efficient diversification and specialization, highreturn indispensable partnerships, ruthless competition elimination, and prudent saving for lean times (cf. 33). These scaled adaptations of Dictyostellids phylogenetically predate socioeconomics-driven change in animal ultrasocieties, strengthening Rauch et al.'s (20) public goods arguments for fungus sociality as well as revealing an exceptional alternate paradigm to Thutupalli et al.'s (31) C. elegans model and to other animal models useful for investigating ultrasociality and additional kinds of economics-supported social organization and operation.

\section{RATIONALITY OF MICROBIAL CONSUMER DECISIONS ON ULTRASOCIAL TENDANCIES}

That said, convincing application of public goods theory to explain and predict the origins and evolution of social microbial behaviors still needs better elucidation. Martinez-Garcia and Tarnita (21) importantly find and caution that use of dimensionally compacted fitness measures, 


\section{Box 2. Traditional Learning and Rational Winning Behavior in Social Microbes}

Like animals, certain taxa of microbes, especially heterotrich ciliates, have been shown to encode and apply stored information about spatiotemporally sensitive relationships (i.e., associations) between unconditioned and conditioned stimuli, as in classical conditioning, or between responses and consequences (i.e., reinforcers), as in instrumental conditioning, to perfect social decision making, such as the economics-based maneuverings of mates and rivals during paired reproductive events. These associative phenomena, unlike epigenetic processes, represent traditional forms of learning that underpin intuitive social logics and flexible goal-directed social reciprocity. As informal inferential systems used by humans, animals and microbes, intuitive social logics typically fail to conform to the axioms of categorical, propositional and predicate logics and of probability calculus. The fallibility of intuitive logics was initially revealed by the groundbreaking research of Nobel-prize-winning scientists who studied simple strategies called representativeness and availability heuristics in humans. Representativeness heuristics reduce inferential tasks to quick exemplar or similarity comparisons. When traits or events used for judgments are unrepresentative or provide only indefinite dimensions for categorization, such as level of mating fitness and availability, the heuristic may become a nonnormative guide to decision making. Availability heuristics employ readily accessible information to judge the frequency, probability and causality of traits and events. Because decisions result from the accessibility of perceived and/or retrieved information, these simple rules of thumb may be corrupted by inaccurate trait or event base rates under the control of subjective factors. Under such conditions, like representativeness heuristics, availability heuristics become a nonnormative guide to decision making.

such as reproductive success, can lead to paradoxical experimental results and spurious conclusions about the sociality of microbes. They cite, as example, the observed occurrence of reproductive skew in wild and laboratory $D$. discoideum chimera. Reproductive skew in the densities of these cellular slime mold subpopulations is typically attributed to social dominance higherarchies caused by strain conflicts over group viability and limited resource availability. However, because chimera populations show fair coexistence and heterogeneity in natural settings, rather than predictable conflict-induced progressive loss of species-wide genetic diversity, some scientists now believe: 1) many, if not all, instances of microbial sociality are illusory and 2) reproductive skew is instead an outcome of life-history tradeoffs and nonsocial multiple fitness traits selected by deterministic and/or stochastic eco-evolutionary pressures. These kinds of experimental findings and interpretations caste doubt on the complexity and rationality or purposefulness of microbial social behavior (cf. 3,11,13-16,18-20,27). To help resolves some of these important issues, improved identification of nonassociatively and/or associatively learned social interactions, as opposed to the mostly genetic and epigenetic components now under widespread investigation, should be stressed.

Traditional forms of learning (Box 2) play significant roles in the adaptation and cultural transmission of traits linked with ultrasocial animals $(4,5,29,32)$. Recent theories (17) are not unique on this matter and one may also expect similar levels of contribution from learning and complex heritable traits in microbial ultrasociality (cf. 6-14,18,20). Thus, inclusion of certain microbes, such as learning-capable Dictyostelids (cf. 28), within the boundary of public-goods- 
dependent ultrasociality will only help shape and advance taxonomically valid sociobiological theory otherwise unachievable with strictly animal-centric interpretations of ultrasocial form and deed. The instance of Dictyostelids learning to switch their economic approach to farming, between largely unregulated public goods and herd-dog-secured private goods models, over lifespans and across generations underscores the need to move beyond animal-centric interpretations of sociobiology and to better evaluate the conserved relationship of traditional learned and inherited goal-direct behaviors with marketplace-influenced organismal adaptation, health, longevity, and life histories. Yet learning in social microbe contexts is best understood in protozoa (6-14) and bacteria (18,20), leaving the need to further refine different taxonomic models of ultrasociality with respect to learned reliability, accuracy, speed, and application of social information processing. Models, such as those involving Dictyostelids, nevertheless offer great potential for unprecedented fuller analysis and understanding

\section{CONCLUSIONS}

Recent theory on the origins of ultrasociality perpetuates the notion that only certain animal clades evolved ultrasocial form and deed, largely because of societal transitions to agriculture and farming-based economic systems (17). In contrast to this animal-centric perspective, extant microbial communities capable of economics-driven primitive farming also fulfill many characteristics of ultrasociality, such as global biomass proliferation, niche diversity and dominance, sophisticated division of labor, physically and communally organized groups, resource management, and social conflict instigation and mediation. Emergence, transfer, and modification of ultrasocial traits in microbial communities, very counter to Martinez-Garcia and Tarnita's (21) assertions about illusory social behavior in D. discoideum, seem to depend on adaptive processes analogous to the multilevel genetic and culture contributions of animal ultrasocieties and may provide exceptional models to investigate the convergent evolution and resilience/stability of economy-favoring ultrasociality within and across taxonomic boundaries and generations of life. That said, Martinez-Garcia and Tarnita's findings raise concerns that apparent ultrasocial tendencies of microbes may be erroneously interpreted as such, emphasizing the need to use similar rigorous scientific methods to more carefully scrutinize any further claims. Such work should also examine the contribution of learning and social logics in effecting winning and/or losing outcomes that benefit an individual microbe and groups of microbes and the impact that these kinds of outcomes have on short- to long-term social structures and processes. Even a putative crude version of ultrasociality and its economics affords microbes a complex, flexible capacity for driving significant environmental changes capable of widespread eco-evolutionary modification of worlds, extinct and extant livable worlds that Earthlings may now or soon explore through man and unmanned space missions. Future astrobiological research should endeavor to understand better the sociobiology of microbes in anticipation and support of potential extraterrestrial life detection.

\section{REFERENCES}

1) Archetti M., Scheuring I., Hoffman M., Frederickson M.E., Pierce N.E., Yu D.W. (2011). Economic game theory for mutualism and cooperation. Ecologyl Letters, 14(12), 1300-1312. 
2) Be’er A., Florin E.-L., Swinney H.L., Payne S.M. (2011). Surviving bacterial sibling rivalry: Inducible and reversible phenotypic switching in Paenibacillus dendritiformis. mBio 2: e00069-11.

3) Ben-Jacob E., Becker I., Shapira Y., Levine H. (2004). Bacterial linguistic communication and social intelligence. Trends in Microbiology, 12, 366-372.

4) Brock D.A., Douglas T.E., Queller D.C., Strassmann J.E. (2011). Primitve agriculture in a social amoeba. Nature 469: 393-396.

5) Brock D.A., Read S., Bozhchenko A., Queller D.C., Strassmann J.E. (2013). Social amoeba farmers carry defensive symbionts to protect and privatize their crops. Nature Communications, 4, 2385.

6) Clark K.B. (2010). Origins of learned reciprocity in solitary ciliates searching grouped 'courting' assurances at quantum efficiencies. BioSystems, 99, 27-41.

7) Clark K.B. (2010). On classical and quantum error-correction in ciliate mate selection. Communicative and Integrative Biology, 3(4), 374-378.

8) Clark, K.B. (2010). Arrhenius-kinetics evidence for quantum tunneling in microbial "social” decision rates. Communicative \& Integrative Biology, 3(6), 540-544.

9) Clark, K.B. (2012). Social biases determine spatiotemporal sparseness of ciliate mating heuristics. Communicative \& Integrative Biology, 5(1), 3-11.

10) Clark K.B. (2013). Ciliates learn to diagnose and correct classical error syndromes in mating strategies. Frontiers in Microbiology, 4, 229.

11) Clark, K.B. (2013). The mating judgments of microbes. In K.B. Clark (Ed.), Social learning theory: Phylogenetic considerations across animal, plant, and microbial taxa, pp. 173-200. Hauppauge: Nova Science Publishers, Inc. ISBN 978-1-62618-268-4.

12) Clark, K.B. (2013). Ciliates learn to diagnose and correct classical error syndromes in mating strategies. Frontiers in Microbiology, 4, 229.

13) Clark, K.B. (2015). Insight and analysis problem solving in microbes to machines. Progress in Biophysics and Molecular Biology, 119, 183-193.

14) Clark, K.B. (2019). Unpredictable homeodynamic and ambient constraints on irrational decision making of aneural and neural foragers. Behavioral and Brain Sciences, 42, e40.

15) Crespi B.J. (2001). The evolution of social behavior in microorganisms. Trends in Ecology and Evolution, 16, 178-183.

16) Dunny G.M., Brickman T.J., Dworkin M. (2008). Multicellular behavior in bacteria: communication, cooperation, competition and cheating. BioEssays, 30(4), 296-298.

17) Gowdy J., Krall L.. (2016). The economic origins of ultrasociality. Behavioral and Brain Sciences, 39, e92.

18) Hellingwerf K.J. (2005). Bacterial observations: A rudimentary form of intelligence? Trends in Microbiology, 13, 152-158.

19) Kaiser D. (2013). Are myxobacteria intelligent? Frontiers in Microbiology, 4, 335.

20) Lyon P. (2015). The cognitive cell: Bacterial behavior reconsidered. Frontiers in Microbiology, 6, 264.

21) Martinez-Garcia R., Tarnita C.E. (2016). Lack of ecological and life history context can create the illusion of social interactions in Dictyostellium discoideum. PLOS Computational Biology, 12(12), e1005246.

22) Michod R.E. (2007). Evolution of individuality during the transition from unicellular to multicellular life. Proceedings of the National Academy of Sciences USA, 104, 8613-8618. 
23) Rainey P.B., Desprat N., Driscoll W.W., Zhang X.-X. (2014). Microbes are not bound by sociobiology: Response to Kümmerli and Ross-Gillespie (2013). Evolution, 68(110), 33443355.

24) Rauch J., Kondev J., Sanchez A. (2017). Cooperators trade off ecological resilience and evolutionary stability in public goods games. Journal of the Royal Society Interface, 14(127), 20160967.

25) Richerson P., Boyd R. (1998). The Evolution of human ultra-sociality. In I. Eibl-Eibisfeldt and F. Salter (Eds.), Ideology, Warfare, and Indoctrinability, pp. 71-95. Berghan Books.

26) Ross-Gillespie A., Kümmerli R. (2014). Collective decision-making in microbes. Frontiers in Microbiology, 5, 54.

27) Shapiro J.A. (1998). Thinking about bacterial populations as multicellular organisms. Annual Review in Microbiology, 52, 81-104.

28) Skoge M., Yue H., Erickstad M., Bae A., Levine H., Groisman A., Loomis W.F., Rappel W.J. (2014). Cellular memory in eukaryotic chemotaxis. Proceedings of the National Academy of Sciences USA, 111(40), 14448-14453.

29) Stallforth P., Brock D.A., Cantley A.M., Tian X., Queller D.C., Strassmann J.E., Clardy J. (2013). A bacterial symbiont is converted from an inedible producer of beneficial molecules into food by a single mutation in the gacA gene. Proceedings of the National Academy of Sciences USA, 110(36), 14528-14533.

30) Tarnita C.E. (2017). The ecology and evolution of social behaviors in microbes. Journal of Experimental Biology, 220,18-24.

31) Thutupalli S., Uppaluri S., Constable G.W.A., Levin S.A., Stone H.A., Tarnita C.E., Brangwynne C.P. (2017). Farming and public goods production in Caenorhabditis elegans populations. Proceedings of the National Academy of Sciences USA, 114(9), 2289-2294.

32) Velicer G.J., Vos M. (2009). Sociobiology of the myxobacteria. Annual Review of Microbiology, 63, 599-623.

33) Werner G.D.A., Strassmann J.E., Ivens A.B.F., Engelmoer D.J.P., Verbruggen E., Queller D.C., Noë R., Johnson N.C., Hammerstein P., Kiers E.T. (2014). Evolution of microbial markets. Proceedings of the National Academy of Sciences USA, 111(4), 1237-1244. 